\title{
The contribution of sleep problems to academic and psychosocial functioning
}

\section{Citation}

Perfect, Michelle M., Deborah Levine\#Donnerstein, Kristen Archbold, James L. Goodwin, and Stuart F. Quan. "The Contribution of Sleep Problems to Academic and Psychosocial Functioning." Psychology in the Schools 51, no. 3 (2014): 273-95.

\section{Permanent link}

http://nrs.harvard.edu/urn-3:HUL.InstRepos:38034709

\section{Terms of Use}

This article was downloaded from Harvard University's DASH repository, and is made available under the terms and conditions applicable to Other Posted Material, as set forth at http:// nrs.harvard.edu/urn-3:HUL.InstRepos:dash.current.terms-of-use\#LAA

\section{Share Your Story}

The Harvard community has made this article openly available.

Please share how this access benefits you. Submit a story.

Accessibility 
The Contribution of Sleep Problems to Academic and Psychosocial Functioning 


\begin{abstract}
The current study examined the concurrent and longitudinal relations among sleep problems with academic and psychosocial functioning in a prospective cohort study, the Tucson Children's Assessment of Sleep Apnea study (TuCASA). Children were assessed between the ages of 6 and 11 years and again approximately five years later. Sleep disordered breathing was assessed via polysomnography and sleep duration, sleep consistency, excessive daytime sleepiness (EDS), and insomnia symptoms were evaluated via parental and self-report. Although regression models for sleep problems yielded minimum effect sizes in predicting standardized achievement scores, they jointly related to lower parent-reported grades and adolescent-reported school problems. Additionally, hierarchical multiple regression revealed that sleep parameters significantly predicted measures of psychosocial functioning with medium (Behavior Assessment Scales (BASC-2) Parent Report Form (PRF)-Behavioral Symptoms Index, Internalizing Symptoms Composite, Self-Report (SRP) Emotional Symptoms Index, Internalizing Composite, Personal Adjustment) to small-medium effect sizes (BASC-2 PRF Externalizing, Adaptive Composites, BASC-2 SRP Inattention/Hyperactivity Composite) above and beyond sociodemographics and IQ. Similar findings occurred for BASC-2 subscales. Parentreported current EDS and youth-reported insomnia symptoms were the most consistent contributors. School psychologists should screen for and treat or make referrals for sleep problems, taking into account chronicity and multiple informants' perspectives, to maximize academic intervention benefits.
\end{abstract}

Keywords: Sleep, academic functioning, social-emotional behavior 


\section{The Contribution of Sleep Problems to Academic and Psychosocial Functioning}

Sleep problems may contribute to adverse school and psychosocial functioning as indicated by special education referrals and status (Luginbuehl, Bradley-Klug, Ferron, Anderson, \& Benbadis,2008) or performance on psychoeducational evaluations (Buckhalt, Wolfson, \& ElSheikh, 2009). A thorough assessment of sleep is not currently part of routine school-wide screenings or comprehensive assessments. Therefore, the potential contributions of inadequate sleep or the relations of sleep disorders, to poor school performance, may not be identified. Thus, school psychologists need to be aware of several aspects of sleep-wake behavior problems that may contribute to poorer outcomes for children and adolescents. The purpose of the present study was to determine if current and long-term sleep-related problems among children, including short sleep duration, inconsistent weeknight and weekend bed and wake times and length, excessive daytime sleepiness (EDS), insomnia symptoms, and sleep-disordered breathing (SDB) were predictive of performance on standardized achievement subtests, parent-reported grades, and parent- and self-behavioral ratings. Most studies have presented a cross-sectional perspective with only a few sleep parameters and/or with younger samples (Buckhalt, El-Sheikh, \& Keller, 2007; Buckhalt, El-Sheikh, Keller, \& Kelly, 2009; El-Sheikh, Buckhalt, Keller, Cummings, \& Acebo, 2007; El-Sheikh, Kelly, Buckhalt, \& Hinnant, 2010; Mayes, Calhoun, Bixler, \& Vgontzas, 2008; Roberts, Roberts, \& Chen, 2002; Roberts, Roberts, \& Duong, 2008), whereas this study collected data on multiple sleep problems at two timepoints, when youth were between the ages of 6 and 11 years and then again, on average 4.7 years later (range 2.90-7.20 years), when youth were between the ages of 9.93 to 17.87 years.

\section{Sleep Problems}

Sleep problems can be viewed along a continuum ranging from insufficient sleep and/or 
daytime sleepiness to sleep disorders, such as SDB and insomnia (El-Sheikh et al., 2010; Gomez, Newman-Smith, Breslin, \& Bootzin, 2010; Gregory \& Sadeh, 2012). The following section describes the research findings related to specific areas of sleep problems.

\section{Insufficient Sleep}

Although individual differences exist in need, the consensus of most professionals is that school-age youth need approximately nine hours of sleep per night (Moore et al., 2009; Pasch, Laska, Lytle, \& Moe, 2010; Roberts, Roberts, \& Duong, 2009). Many youth do not sleep the recommended number of hours and up to $80 \%$ of adolescents are chronically sleep deprived (Pasch et al., 2010). The clinical consequences of not getting enough sleep may include disrupting the processing of obtaining new knowledge, affecting school-related outcomes (Dewald, Meijer, Oort, Kerkof, \& Bogels, 2010; Gomez et al., 2010), and diminishing the ability of the brain to consolidate and strengthen material learned during the day (Walker, 2009). Further, sleep loss results in emotion dysregulation and consequent psychological maladjustment (Gregory \& Sadeh, 2012) through disrupting connections between the prefrontal cortex and the amygdala (Yoo, Gujar, Hu, Jolesz, \& Walker, 2007). Regarding the impact of sleep on performance in school, total sleep time (TST) has been associated with more school problems and lower grades (Fredriksen, Rhodes, Reddy, \& Way, 2004; Roberts et al., 2009). For instance, Wolfson and Carskadon (1998) found that students who reported sleeping almost a half hour less than their classmates were more likely to report having grades of $\mathrm{C}$ or lower. Studies have also reported short (Fredriksen et al., 2004; Roberts et al., 2009) and long-term (Fredricksen et al., 2004) associations between chronic sleep debt and depressive symptoms and low levels of selfesteem. In the Tucson Children's Assessment of Sleep Apnea (TuCASA) study, children between 6 and 11 years of age who slept less than 7.5 hours during home polysomnography 
(objective measure of sleep architecture, time, and breathing), had a greater prevalence of learning problems, anxiety, and depression five years later (Silva et al., 2011), in comparison to those who slept more than 9 hours. Other studies (Gregory \& Sadeh, 2012; Pasch et al., 2011; Wolfson \& Carksadon, 1998) also reported that short sleep duration on school nights associated with depressive symptoms and behavioral difficulties. In contrast, Moore and colleagues (2009) did not find that sleep duration measured using an actigraphy (watch-size device that estimates sleep-wake activity) related to self, parent, or teacher reports of internalizing or externalizing symptoms among adolescents.

\section{Inconsistent Sleep Patterns}

Although the amount of sleep needed does not change significantly into adolescence, the timing of sleep onset shifts later. Despite going to sleep later, youth usually have to wake up prematurely because of early school start times, resulting in insufficient sleep. To compensate for the loss of sleep during the week, adolescents may wake up later on the weekends (Moore et al., 2009). These sleep patterns pose two difficulties. First, diminished academic performance is associated with self-reported later bedtimes (Wolfson \& Carskadon, 1998), as well as a discrepancy between self-reported bed and wake times on non-school nights compared to a school night (O’Brien \& Mindell, 2005; Wolfson \& Carskadon, 1998). Second, the inconsistent sleep and bedtimes between school and non-school nights may also increase risk-taking behaviors (e.g., sexual behaviors, tobacco use, and safety behaviors; O'Brien \& Mindell, 2005; Pasch et al., 2011). Difficulties inhibiting inappropriate responses or delaying gratification may be due to the brain-behavior relationship (i.e., pre-frontal cortex involvement) that underlies both sensation-seeking behaviors and sleep (Dahl, 1996). The association between depressive symptoms and later bedtimes or longer weekend sleep duration is equivocal (Pasch et al., 2011; 
Wolfson \& Carskadon, 1998), though females may be more affected (Wolfson \& Carskadon, 1998).

\section{Daytime Sleepiness}

One-fifth to half of youth report feeling sleepy during the day (Dewald et al., 2010) as they struggle to stay awake or actually fall asleep during daytime activities. Research has supported a link between self-reported sleepiness and lower school performance (Dewald et al., 2010; Gibson et al., 2006; Joo et al., 2005; Pagel, Forister, \& Kwiatkowski, 2007; Pagel \& Kwiatkowski, 2010; Perez-Chada et al., 2007; Perfect et al., 2012; Shin, Kim, Lee, Ahn, \& Joo, 2003) as well as self-reported depression and anxiety in adolescents (Moore et al., 2009).

\section{Insomnia Symptoms}

Insomnia is characterized by difficulties initiating and maintaining sleep. The prevalence in children and adolescents ranges from $1 \%$ to $6 \%$, with considerably higher estimates for children with mental health or developmental difficulties (Mindell et al., 2006; Roberts et al., 2008). Although the clinical diagnosis of insomnia and its school-related outcomes rarely have been examined in youth, studies based in the United States and other countries found that adolescents and young adults who experienced various insomnia symptoms had lower GPAs (Chung, Kan, \& Yeung, 2011; Gaultney, 2010; Lazaratou, Dikeos, Anagnostopoulos, Sbokou, \& Soldatos, 2005; Pagel \& Kwiatkowski, 2010) and were more likely to fail a grade in school (Kahn et al., 1989). Further, Roberts et al. (2002) conducted a large longitudinal study of preadolescents and adolescents and found that insomnia symptoms predicted self-esteem and depression one year later. Similarly, among adolescents, insomnia that persisted over time was associated with somatic complaints, social challenges, mental health difficulties, and interference in school and work activities (Roberts et al., 2008). 


\section{Sleep-Disordered Breathing}

Depending on the criteria for sleep-disordered breathing (SDB) used in previous studies and age of the child, SDB affects between $3 \%$ and $23 \%$ of youth (Goodwin, Vasquez, Silva, \& Quan, 2010; O’Brien et al., 2004). SDB is diagnosed when breathing is occluded or nearly occluded in the upper airway for short segments of time during sleep. Obstructive sleep apnea (OSA), defined when breathing completely stops, is the most severe form of SDB. Beebe, Ris, Kramer, Long, and Amin (2010) recently found that, relative to those without SDB, students with SDB had poorer grades, and teacher-reported learning problems, but no differences occurred in performance on neurocognitive assessments. The authors concluded that the negative effects of SDB might directly impact ecologically-valid measures of school performance, but not emerge on standardized assessments. Their explanation was that standardized achievement tests assess stored information, whereas grades are based on the students' ability to acquire new knowledge and engage in behaviors (e.g., studying) that increase the likelihood that they will retain the new material. In the first examination of TuCASA (Goodwin et al., 2003), and in a more recent article from this cohort, current SDB was related to parental reports of learning problems (Silva et al., 2011). Yet in a subsample of TuCASA participants, Kaemingk and colleagues (2003) did not find differences between those with and those without SDB on reading, writing, and math achievement standardized tests. Comparing youth with a respiratory disturbance index (RDI) in the top $15 \%$ relative to youth in the bottom $85 \%$, Mulvaney et al. (2006) found that parents reported more cognitive problems as measured by the Conner's Parent Rating Scale-Revised (CPRS-Revised).

With regard to psychosocial functioning, Mulvaney et al. (2006) found at-risk or clinically elevated scores on the CPRS-Revised and the Child Behavior Checklist in the areas of 
attention, aggressivity, oppositionality, and social problems. Zhao, Sherrill, Goodwin, and Quan (2008) reported that SDB was associated with social problems and somatic complaints. Beebe, Ris, et al. (2010) found that teachers of youth who had SDB reported decreased attentiveness, whereas parents of youth with SDB reported youth to be more hyperactive, anxious, depressed, and inattentive. Nonetheless, in the aforementioned studies on SDB and outcomes, other sleep parameters were not considered, and thus examining the combination of other sleep factors may help us better understand the consequences of sleep problems on school and social-emotional outcomes.

\section{Multiple Sleep Problems and Longitudinal Findings}

Most studies have focused on one type of sleep disturbance (e.g., short sleep duration) or may have measured more than one parameter, but not examined them in combination in the same model. In a cross-sectional study, Mayes et al. (2008) examined 14 sleep variables assessed with polysomnography (PSG) and parental reports of their children's sleep problems (ages 6 to 12), in relation to standardized achievement test scores, having a diagnosis of a learning disability, and parent-reported learning problems among children in elementary school. In this cross-sectional study, the researchers found that only parental-reported sleep disturbances related to academic functioning, but the differences diminished after controlling for scores on a measure of Attention Deficit Hyperactivity Disorder (ADHD).

El-Sheikh, Buckhalt and colleagues (Buckhalt et al. 2007; Buckhalt et al. 2009; ElSheikh et al., 2007; El-Sheikh et al., 2010) examined students who were in third grade and then again two years later when they were in fifth grade. Self-reported sleep problems at time1 were associated with poorer performance on the Stanford Achievement Test (SAT) Language and Math subtests and the Verbal and Nonverbal subtests Otis-Lennon School Ability Test (El- 
Sheikh et al., 2007). However, mothers with less education, lower socioeconomic status, and African-American race moderated many of the relations between children's sleep duration and self-reported poor sleep habits with achievement (Buckhalt et al., 2009; El-Sheikh et al., 2007). Further, objectively-measured variability in sleep onset and wake times related to SAT Math and Language subtest scores (Buckhalt et al., 2009). Buckhalt et al. (2009) also reported that greater daytime sleepiness was associated with lower scores on the SAT Mathematics, Language, and Reading subtests. El-Sheikh et al. (2010) reported that parents' reports of children's sleep problems at time 1 were associated with social-emotional difficulties at time2. Sleep-wake behavior problems and sleepiness, measured via self-report, also impacted the display of internalizing problems (parent and child perceptions) and parent-reported externalizing problems in students in elementary school. In contrast, the longitudinal and cross-sectional analyses did not find associations with objectively-measured irregular sleep patterns and internalizing and externalizing symptoms (El-Sheikh et al., 2010). More recently in a different and larger sample, this research team (Bub, Buckhalt, \& El-Sheikh, 2011) found that youth who felt sleepy starting in third grade and continued to feel sleepy at two later timepoints, were less likely to exhibit improved verbal comprehension skills.

\section{Overview of Current Study}

The current study examined several sleep parameters and disturbances (i.e., duration; consistency of night and weekend sleep times; EDS; insomnia symptoms; and SDB) in relation to parental reports of children's grades and performance on select subtests of the Woodcock Johnson Tests of Achievement-Third Edition (Woodcock, McGrew, \& Mather, 2001), as indices of academic functioning, and subscales from the Behavior Assessment System for Children-2 ${ }^{\text {nd }}$ Edition Parent Report Form and Self-Report (BASC-2 PRF and SRP; Reynolds \& Kamphus, 
2004) as measures of psychosocial functioning. The sleep variables were collected at two timepoints, when youth were elementary-school age and then when they were in the preadolescent to adolescent age range, allowing us to examine concurrent sleep disturbances and functioning, as well as sleep problems over time in relation to academic and psychosocial functioning. The following hypothesis guided our analyses: Current sleep problems, individually and in combination, would significantly relate to current academic and psychosocial functioning. In addition, we believed that time2 sleep variables would be better predictors (i.e., contribute significant variation) than timel variables, but that previous and current problems (i.e., both time1 and time 2 variables adding unique variation for a particular sleep problem) also would be indicative of poorer academic achievement and problematic behaviors. To determine the contribution of sleep in predicting separate measures of achievement and adjustment above and beyond sociodemographics and IQ, we entered verbal and non-verbal IQ scores, body mass index (BMI), age, sex, race/ethnicity (Hispanic or non-Hispanic), parent's highest education level, and income as the first step in all hierarchical regression models.

\section{Methods}

\section{Participants}

The participants from this study were part of a larger sample derived from the TuCASA study, which prospectively examined Hispanic and Caucasian children between the ages of 6 to 11 years of age to determine the prevalence and incidence of SDB and its effects on behavioral indicators on children who were school-age but pre-pubertal. During the initial recruitment for a cross-sectional study, participants were identified using a screening questionnaire at the largest school district in the Southwestern region of Arizona, with 503 participating in the initial examination (time1). The study's original exclusion criteria included impairments that would 
impact performance on neurobehavioral measures or interfere with sleep. Approximately five years later (mean 56.4 months), additional funding provided an opportunity to add a longitudinal component (time2) to the study to collect data on the same participants. Eligibility for the second study was limited to families who participated in the first examination (Goodwin et al., 2001; Silva et al., 2011)

Three hundred and forty-eight 9.93 to 17.87 year-old participants of the $503(69.18 \%)$ completed various assessments at time2. Three hundred and nineteen participants had a valid PSG, all with completed parent and child sleep habit questionnaires (SHQ), and 273 completed neurobehavioral measures, 10 of whom did not undergo a PSG. For purposes of this study, we included a select subsample of 263 youth $(52.29 \%$ of the original 503$)$ who completed the PSG, SHQ, and neurobehavioral data at both timepoints. Two hundred and thirty-nine parents completed the parental report of his/her child's behavior, with 227 (86.3\% of the 263 and $45.1 \%$ of the original 503) having both parent and child data. No significant differences were evident between participants with completed child data at time 2 and those without completed data, regarding sex, age, BMI, or parental-reported sleep variables at time 1 or 2 . However, there was a trend for youth who participated to be younger in comparison to those not included in the time 2 sample. As reported previously (Silva et al., 2011), a higher attrition existed among families of Hispanic origin. Additionally, participants who participated at time2 also had higher IQ scores at the first examination than those who did not re-enroll. Cases without parent behavioral data were significantly more likely to be Hispanic, have lower IQs, and be younger than those with parent behavioral data. However, they did not differ on the other sociodemographic variables or Timel parental-reported sleep variables. Among those who participated, parents who identified as 
Caucasian were more likely to have graduate degrees and report the higher income brackets than would be expected by chance.

\section{Measures}

A variety of measures of sleep and neurobehavioral functioning were administered to participants at both timepoints. We present the ones measuring academic and psychosocial functioning that were most relevant to this particular study. In some cases, measures were added or removed during the second examination to perform an efficient, yet informative neurocognitive battery with measures that were not previously available during the first administration or validated for the age range studied. Parents reported on their child's sleep during both timepoints. However, because of participants' young age and given the difficulty in assessing certain sleep problems on oneself, youth were not asked to report their sleep during the first examination, but they were asked in the second examination.

Home-Based Polysomnography (PSG). Unattended overnight PSGs were completed with the Compumedics PS-2 system (Abbotsford, Victoria, Australia). For this study, participants were allowed to sleep for their usual sleep period. We recorded central channels of the electroencephalogram (EEG; $\mathrm{C}_{3} / \mathrm{A}_{2}$ and $\left.\mathrm{C}_{4} / \mathrm{A}_{1}\right)$, bilateral electrooculogram (EOG), a bipolar submental electromyogram (EMG), and electrocardiogram (ECG). SDB was evaluated using channels that measure thoracic and abdomen movement (inductive plethysmography bands), nasal pressure, airflow (nasal/oral thermister), and arterial oxygen saturation (finger pulse oximetry; $\mathrm{SaO}_{2}$ ). A trained technician scored the sleep studies in accordance with Rechtschaffen and Kales' (1968) criteria. For SDB, apneas were scored if the amplitude (peak to trough) of the thermister airflow signal decreased to at least $25 \%$, of baseline for more than six seconds or two breath cycles. Hypopneas were scored for events that were not deemed apneas if the amplitude of 
any respiratory signal decreased below $70 \%$ or more of the baseline amplitude. The respiratory disturbance index (RDI) was derived from the mean number of apneas and hypopneas per hour during the time the participant was asleep. As used in previous studies, a RDI $\geq 1$ event/hour associated with a 3\% oxygen desaturation indicated the presence of SDB (Goodwin et al., 2003; Goodwin et al., 2010; Silva et al., 2011).

Sleep Screening and Sleep Habits Questionnaires (SHQs). In the absence of standardized pediatric sleep questionnaires at the start of the initial examination, the SHQ was created to assess parental perceptions of their children's sleep patterns; the questions were administered again at the second examination of the study. During the second examination, youth were asked to self-report their sleep habits as well. Several publications have used items from this questionnaire to capture parent reports of their child's sleep habits and disturbances and found them to be related to clinical outcomes (e.g., Goodwin et al., 2003; Goodwin et al., 2010; Silva et al., 2011). The following questions were used for this study.

Sleep duration. At both timepoints, parents were asked to report how many hours per night their child slept on a typical school night and non-school night. They also wrote in the times when their child usually went to bed and woke up on school night and nonschool nights, respectively. At the second examination, youth also provided this information. We used the written number of hours for school night TST, weekend night TST, and average TST (i.e., the average of school night and weekend night TST; Wolfson \& Carskadon, 1998). One recent study (Beebe, Ris, et al., 2010) used an average of the written times and the computed times (calculated by subtracting wake time from bed time). We elected not to use this methodology because the SHQ asked when the youth went to bed, not when they fell asleep. This wording may lead to an overestimation 
of TST by including time in bed not sleeping (Pasch et al., 2011). Wolfson et al. (2003) found that youth self-reports of typical sleep timing and duration were comparable to objectively-measured sleep over multiple nights, particularly on school nights, suggesting the validity of youths reporting their usual bedtimes.

Another variable derived from TST was the difference between reported weekend-night TST and school-night TST at both timepoints for parents and the second examination for youth (referred to as weekend oversleep; O’Brien \& Mindell, 2005; Wolfson \& Carskadon, 1998). We also determined the discrepancy between reported bedtimes during school nights and non-school nights (referred to as weekend delay; O’Brien \& Mindell, 2005; Wolfson \& Carskadon, 1998).

Excessive Daytime Sleepiness (EDS). A parent and child EDS variable was created, based on whether the parent (at both timepoints) or youth (at time2), endorsed a response of frequently or almost always on any of the following questions: Is your child (are you) sleepy during the daytime?; Does your child (do you) fall asleep at school?; or Does your child (do you) fall asleep while watching television? This variable was used in previous publications (Goodwin et al., 2003; Goodwin et al., 2010; Silva et al., 2011).

Difficulty Initiating and Maintaining Sleep (DIMS): As an indicator of insomnia symptoms, a parent and child DIMS variable was created based on whether the parent (both timepoints) or youth (time2) indicated they still had any of the following problems: trouble falling asleep; trouble staying asleep; and waking up too early and not being able to get back to sleep? (Goodwin et al., 2010).

Woodcock Johnson Tests of Achievement-III (WJ III ACH; Woodcock et al., 2001). The WJ III ACH is a standardized battery of tests of achievement that yields information about 
a student's academic abilities. The subtests available were: Letter-Word Identification (both times), Reading fluency (time2), Math Fluency (time2), and Writing Fluency (time2). Raw scores are converted to standard scores $(M=100, S D=15)$ based on the examinee's age. Letter-Word Identification involves the examinee reading words aloud. Reading Fluency assesses the speed of reading sentences. Math Fluency measures how fast someone can perform simple calculations. Writing Fluency is a timed task in which the respondent is given three words to write sentences as quickly as possible. Normative samples' scores had reliability coefficients ranging from alphas of .88-.94.

Parental reported grades. During the second timepoint, parents were asked: How well does your child do in school? $(1$ = Gets mostly A's or is outstanding; 2 = Gets mostly A's and B's or is excellent; 3 = Gets mostly B's or is very good; $4=$ Gets mostly B's and C's or is good; $5=$ Gets mostly C's or is satisfactory/average; $6=$ Gets mostly grades worse than C's or is below average). Since B's are equivalent to a 3.0, reflecting very good performance, we dichotomized the grade variable to at least B's or better (choices 1 - 3) versus some C's or lower (choices 4 6; Wolfson \& Carskadon, 1998).

The Behavior Assessment System for Children-Second Edition (BASC-2; Reynolds \& Kamphus, 2004). The BASC-2 Parent Rating Scale (PRS) and Self-Report of Personality (SRP) were completed at the second examination and used to assess social-emotional functioning. Parents rate the child's behavior on a four-point Likert scale $(1=$ "Never" to $4=$ "Almost Always"). Some questions on the SRP use the same 4-point Likert scale, whereas others are true/false format. The PRS yielded composite scores in the areas of Behavioral Symptoms (Hyperactivity, Aggression, Depression, Attention Problems, Atypicality, and Withdrawal scales); Adaptive Skills (Adaptability, Social skills), Internalizing Behaviors, and Externalizing 
Behaviors. The normative samples' scores had reliability coefficients ranging from alphas of .70.95.

Additionally, the SRP yields the following composite scores (and related subscales): School Problems (Attitude to School, Attitude to Teachers, and Sensation Seeking, Adolescent version only), Internalizing Problems (Atypicality, Locus of Control, Social Stress, Anxiety, Depression, and Sense of Inadequacy), Inattention/Hyperactivity (Attention Problems and Hyperactivity), Personal Adjustment (Relations with Parents, Interpersonal Relations, SelfEsteem, and Self-Reliance), and Emotional Symptoms (Social Stress, Anxiety, Depression, Sense of Inadequacy, Self-Esteem and Self-Reliance). The normative samples' Cronbach's coefficient alphas for the SRP composites and subscales ranged from .61-.96.

Sociodemographics and IQ. Parents were asked about their child's race/ethnicity, date of birth, income level (time2), and highest level of education (time2). Eleven response choices for income existed in $\$ 5,000$ increment ranges starting with up to $\$ 5,000-\$ 50,000$ or more: Highest level of parents' education was categorized as some high school, high school graduate or equivalent, associate degree or vocational school, some college, 4-year-college graduate, graduate school. Children's height and weight were taken on the night of the PSG at both timepoints; body mass index (BMI) was computed using the Center for Disease Control's computation algorithm and are reported as z-scores and percentiles (CDC, 2012). The Wechsler Abbreviated Scale of Intelligence (WASI; Wechsler, 1999) provided a brief screening measure of intelligence. It contains four subtests (Vocabulary, Block Design, Matrices, and Similarities) that comprise three composite scores: Full Scale IQ score (FSIQ), Verbal IQ (VIQ), and Performance IQ (PIQ). Raw scores are converted to T-scores $(M=50, S D=10)$ for the subtests 
and standard scores for the composites. Reliability coefficients from the normative sample for the FSIQ, VIQ, and PIQ were $.96, .96$, and .94, respectively.

\section{Procedures}

Both the University's and local school district's institutional review boards approved the study. Prior to performing procedures in the study (other than the sleep screening questionnaire that had already been completed), parents provided informed consent, and the child provided assent. On the night of the PSG visit for both timepoints, they completed the SHQ; they also completed the original screening questions and SHQ during the second examination. During the second examination, youth also self-reported the same sleep parameters as their parents. To complete the home-based PSG, research assistants went in pairs to the participants' homes approximately one hour before their typical bedtime. About four-fifths of the sleep studies were conducted on a school night. Within approximately one month of the sleep study, children were administered a variety of neurobehavioral measures that included the BASC-2 and WJ III ACH (Silva et al., 2011).

Data analyses. The analyses focused on the sample of participants who were successfully contacted, consented, and agreed to participate in at least the PSG and neuropsychological evaluation portions of the study. For the BASC-2 PRS subscale outcomes, the analyses included the cases in which a parent completed this measure. Therefore, individual models varied slightly in sample size. To determine how much the combination of sleep variables predicted scores on the WJ III ACH, BASC-2 PRS, and SRP, above and beyond the sociodemographic and IQ variables, we performed a series of hierarchical multiple regression analyses with sociodemographic variables (age, sex, BMI, Verbal IQ, Performance IQ, race/ethnicity, income range) entered in the first block and parent- (time1 and 2) and self- 
(time2) reported sleep variables (EDS, insomnia symptoms, average sleep duration, weekend oversleep, and weekend delay) plus SDB (time1 and time2) entered together in the second block. $\mathrm{R}^{2}$, which is the proportion of variation accounted for in this model, is a measure of effect size; an $\mathrm{R}^{2}$ of .02 is considered a small effect, .13, a medium effect, and .26 or greater a large effect (Cohen, 1988). None of the models showed evidence of multicollinearity as indicated by tolerance values ranging from .55 to .98 . Given that parent-reported grades were dichotomized, we used logistic regression models with significant sociodemographic and sleep variables as the predictors. The effect size estimate for LR was Nagelkerke $\mathrm{R}^{2}$ (Nagelkerke, 1991), which has a range of $0-1$.

\section{Results}

\section{Sleep Problems Over Time}

Table 1 shows the breakdown of sleep problems during the first and second examinations, as reported by the parents (time1 and 2) and youth (time2). Overall, youth reported sleeping an average of 8.92 hours $(S D=1.23)$, with a 1.50 hours $(S D=1.22)$ delay in bedtime on non-school nights; parents reports were not significantly different than their child's at time 2 on these variables. Sixty-eight percent of both parents and youth indicated the youth slept less than the recommended 9 hours on school nights. In comparing sleep duration of school nights and non-school nights and for time 1 and 2 using parent reports, no significant differences existed between sleep length on school nights and non-school nights at time1, $t(262)=-.16, p=$ $.87, d=.02$, and non-school nights between times 1 and $2, t(262)=1.51, p=.13, d=.11$. Parents reported that youth slept less on school nights at time 2 than they did at time $1, t(262)=$ 14.32, $p<.001, d=1.17$. At time2, both parents, $t(262)=-11.07, p<.001, d=.80$, and youth, $\mathrm{t}(263)=-6.14, p<.001, d=.50$, reported that TST was significantly less on school nights than 
non-school nights. Nearly half the youth evidenced at least one sleep disturbance, with insomnia symptoms the most common (51.30\%), followed by EDS (31.20\%) and SDB (16.70\%).

Although significant, the association among child and parent reports on the same sleep variables at time2 yielded small to moderate correlations, with weekend delay, $r(263)=.56, p<.001$, and sleep duration on school nights having the strongest relations $r(263)=.51, p<.001$. The rates of reporting sleep problems were lower for parents than youth (e.g., EDS 14.4\% versus 31.1\%).

\section{School Functioning}

Although various sleep variables significantly predicted WJ III ACH scores (Basic Word Reading, Reading Fluency, Writing Fluency, Math Fluency), they contributed very little (i.e., $1 \%-7.5 \%$ of variation) to the model, after controlling for the sociodemographic and IQ variables. However, when sleep variables were added to the model in predicting students who had grades $\mathrm{C}$

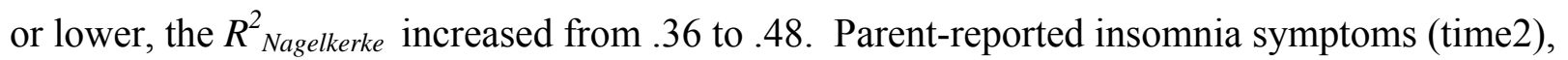
$O R=3.04, p=.02, C I[1.10,8.36]$, and lower self-reported TST, $O R=.91, p=.03, C I[.86,1.0]$, and $\mathrm{EDS}, O R=2.72, p=.03, C I[1.09,6.81]$, increased the likelihood that students would have lower grades. Specifically, youth with lower grades had a mean TST of 8.48 hours $(S D=1.37)$, whereas youth with As and/or Bs had a mean TST of 9.09 hours ( $S D=1.14)$.

As another measure of school functioning, we considered the BASC-2 SRP School Problems Composite and its two subscales. Sleep variables contributed $12.50 \%$ of the variation above and beyond sociodemographic variables in predicting this composite, $R^{2}=.18, F(25$, $251)=8.51, p<.001$, Time2 parent-reported EDS and weekend oversleep, and youth-reported insomnia symptoms added their own unique variance in the model. Additionally, sleep problems contributed $12.4 \%$ of the variation above control variables in predicting both perceived problems in school, $R^{2}=.18, F(25,251)=3.61, p<.001$ and interacting with their teachers, $R^{2}=.20, F(25$, 
$251)=2.29, p=.001$. For the BASC-2 SRP Attitude to School subscale, parent reported sleep duration (time2) and youth reports of insomnia symptoms contributed significantly to the model. Parent-reported weekend oversleep (time2) and youth-reported insomnia symptoms added their own unique variation in predicting the BASC-2 SRP Attitude to Teachers subscale.

\section{Psychosocial Functioning}

The mean of the BASC-2 PRF and SRP scores were within the average range (see Tables 2 and 3). Given the large amount of data, we decided to use the following criteria for reporting. We report in text when sleep variables (step 2) contributed at least small-medium effects $(10 \%)$ of the variation in predicting the criterion (i.e., BASC-2 score) above and beyond sociodemographics and IQ (step 1) in the hierarchical multiple regression analyses. We present additional information in Tables $4-8$ of models for which the sleep variables had at least medium effect sizes $\left(R^{2} \geq .13\right)$. Even after sociodemographic variables and IQ were partialed out, sleep variables contributed $15.60 \%$ of the variation in predicting BASC-2 PRF Behavioral Symptoms Index scores, $R^{2}=.26, F(25,217)=2.70, p<.001$. Parents' reports of EDS (time1) and insomnia symptoms (time2), youth reports of insomnia symptoms, and current SDB added their own unique variance (see Table 4). In terms of the BASC-2 SRP Emotional Symptoms Index, $R^{2}$ $=.29, F(25,251)=3.67, p<.001$, sleep variables contributed $17.3 \%$ of the variation. Parent reported weekend delay (time1) and youth reports of less TST and insomnia symptoms contributed uniquely to the model (see Table 4).

Regarding BASC-2 PRF Internalizing Composite scores, sleep variables explained 17.4\% of the variation, after controlling for sociodemographics and IQ, $R^{2}=.27, F(25,217)=2.95$, $p<.001$. Parents' reports of their child having EDS (time1) and insomnia symptoms (time2) added their own unique variation (see Table 4). Similarly, sleep variables contributed $20 \%$ of the 
variation above and beyond sociodemographics and IQ in predicting the BASC-2 SRP Internalizing Composite, $R^{2}=.31, F(25,251)=4.04, p<.001$. Parents' reports of their child having EDS (time2) and youth reports of EDS and insomnia symptoms contributed uniquely to the model (see Table 5).

Several sleep variables significantly contributed $18.70 \%, 16.60 \%$, and $19.90 \%$ of the variation in predicting the BASC-2 PRF Depression subscale and the BASC-2 SRP Depression and Anxiety subscales (see Tables 5-6). Parent-reported EDS (time1), youth-reported insomnia symptoms, and current SDB added their own unique variation in predicting the BASC-2 PRF Depression subscale, whereas parent-reported EDS (time2), youth-reported insomnia symptoms and sleep duration were still significant after controlling for the other variables in the block when the BASC-2 SRP Depression subscale was the criterion. Self-reported EDS, insomnia symptoms, sleep duration, and weekend delay all added unique variation in predicting the BASC-2 SRP Anxiety subscale. Sleep variables predicted the BASC-2 PRF Anxiety subscale accounting for $12.80 \%$ of the variation above demographics and IQ, $R^{2}=.21, F(25,217)=2.03, p=.004$; parentreported insomnia symptoms (time2) and self-reported sleep duration added their own unique variation.

Sleep variables also significantly related to the BASC-2 SRP Atypicality subscale $(10.00 \%), R^{2}=.21, F(25,251)=2.34, p=.001$, with the parent EDS (time2) and youth insomnia contributing significantly. Parent-reported insomnia symptoms (time2) and youth-reported weekend oversleep uniquely contributed to the BASC-2 PRF Atypicality subscale; sleep variables contributed $15.40 \%$ of the variation above demographics and IQ (see Table 4 ). Above and beyond demographics and IQ the sleep variables contributed $12.90 \%$ of the variation in predicting the BASC-2 PRF Withdrawal subscale, $R^{2}=.19, F(25,217)=1.77, p=.017$, with parent 
reported sleep duration (time2) youth reported insomnia, and current SDB remaining significant after controlling for the other variables in the block. Data for the BASC-2 PRF Somatization subscale are depicted in Table 7, with parent-reported insomnia symptoms (time2) and youth reported weekend oversleep adding their own unique variation. The sleep variables contributed medium effects $(13.00 \%)$ to the BASC-2 SRP Personal Adjustment Composite, $R^{2}=.22, F(25$, $251)=2.48, p<.001$. Parents' reports of their child going to bed later on weekends (time1) and youth reports of insomnia symptoms added their own unique variation (see Table 6). Youth reported insomnia was the only significant sleep variable $12.5 \%$ in predicting the BASC-2 SRP Relations with Parents, $R^{2}=.18, F(25,251)=1.95, p=.006$, and the Locus of Control $(15.40 \%)$, $R^{2}=.23, F(25,251)=2.67, p<.001$, subscales (see Table 7 for Locus of Control). The sleep variables had at least medium effects in predicting the BASC-2 SRP Self Esteem (14.00\%), Social Stress (15.00\%), and Sense of Inadequacy (15.70\%) subscales (see Tables 7 and 8 ). The BASC-2 SRP Interpersonal Relations and Self-Reliance subscales were not predicted by sleep variables by at least $10.00 \%$.

Sleep variables significantly contributed $12.2 \%$ of the variability to the BASC-2 PRF Externalizing Composite, $R^{2}=.22, F(25,217)=2.16, p=.002$. Parents' reports of their child having EDS (time1) and youth reports of insomnia symptoms added their own unique variance. Within this composite, the BASC-2 PRF Hyperactivity subscale was significantly predicted by sleep variables $(12.9 \%), R^{2}=.25, F(25,217)=2.53 p<.001$. Parent reported EDS (time1) and insomnia symptoms (time2) added their own unique variation. Although sleep problems did not contribute at least $10 \%$ of the variation in predicting the BASC-2 PRF Aggression subscale, they did predict the BASC-2 PRF Conduct Problems subscale $(11.90 \%), R^{2}=.20, F(25,217)=1.95 p=.006$. Parent reported EDS (time1) and youth reported insomnia contributed significantly to the model. The 
BASC-2 SRP Hyperactivity/Inattention Composite, $R^{2}=.19, F(25,251)=2.21, p=.001$, was significantly predicted by the sociodemographic and sleep variables $(12.00 \%$ variation accounted for), with youth reports of insomnia symptoms uniquely contributing. Sleep problems jointly contributed $11.30 \%$ and $11.40 \%$ to the BASC-2 SRP Attention, $R^{2}=.21, F(25,251)=2.43 p<.001$, and Hyperactivity, $R^{2}=.16, F(25,251)=1.76, p=.017$, subscales, respectively. Youth reported insomnia was the only sleep variable uniquely predicting both of those subscales.

Sleep variables accounted for $10.00 \%$ of the variation in predicting the BASC-2 PRF Adaptive Behavior Composite, after controlling for sociodemographics, $R^{2}=.30, F(25$, $217)=3.34, p<.001$. Only current SDB added its own unique variation. Sleep variables did not contribute more than $10.00 \%$ of the variation in the regression models for the subscales on this composite.

\section{Discussion}

The present study examined the relative contribution of sleep problems in predicting academic and psychosocial functioning in youth between the ages of 9 and 17. Overall, the findings supported that sleep problems jointly predicted lower grades, youth's perceptions that they were struggling in school, and negative attitudes toward teachers and school. Consistent with some studies (Beebe, Ris et al., 2010; Mayes et al., 2008), but in contrast to El-Sheikh, Buckhalt and colleagues (Buckhalt et al., 2007; Buckhalt, El-Sheikh, et al., 2009; El-Sheikh et al., 2007), sleep problems contributed little variance in predicting student performance when standardized achievement test scores were the criterion. However, these latter investigators studied a younger sample of participants of whom more than one-third were African American. On the other hand, sleep variables jointly yielded small-medium effects sizes (i.e., contributed at least $10 \%$ of the variation in a model) for all but three of the BASC-PRF and two of the SRP 
subscales. In fact, medium effect sizes were found in many the majority of the models. Accordingly, multiple sleep problems additively related to parent-reported behavioral problems, youth-reported emotional problems, parent- and youth-reported internalizing symptoms including depressive symptoms and youth-reported anxiety, parent-reported physical stress and health complaints and unusual behaviors and thinking, and youth-reported external locus of control, adjustment difficulties, low self-esteem, stress in social situations, and perceptions of inadequacy.

\section{Insufficient Sleep}

When averaging parent and student reports of hours slept across the whole week, the current sample mean TST is 9.13 and 8.92, respectively. This average sleep duration is consistent with the recommended nine hours of night for optimal sleep (Pasch et al., 2010; Roberts et al., 2009). However, over two-thirds of adolescents experienced sleep debt on school nights $(<9$ hours). Further, consistent with the findings of Wolfson and Carskadon (1998), we also found that students with grades of $\mathrm{C}$ or lower slept 30 minutes less than those who earned As or Bs. This half hour puts them under the recommended nine hours, further supporting the need for all youth to sleep that long. Additionally, parent-reported sleep duration significantly predicted youth's negative perceptions of school. Future studies should examine what is the 'optimal' sleep time to achieve maximum potential in achievement outcomes, taking into account individual differences in sleep need.

Average sleep duration, particularly youth-reported, remained significant even after controlling for sociodemographics and IQ in the first block and for other sleep problems in the second block in several models. In particular, youth who reported shorter TST also reported experiencing more emotional difficulties, higher levels of anxiety, depressive symptoms, and 
feelings of inadequacy. Less self-reported TST also significantly related to parents' reports of their child's anxiety. Parental-reported sleep duration was associated with their perceptions that their child tends to isolate him or herself. Although we did examine TST on school nights and non-school nights separately, in most cases, average sleep duration also was significantly related to the criterion as well. We also focused on subjectively measured sleep duration because selfreport is more feasible in school psychological practice. However, it is important to keep in mind that our measurement was based on an estimate of the child or parent rather than a computed TST or over multiple nights. Nonetheless, these findings provide further evidence that youth who are not getting sufficient sleep are at-risk for behavioral and emotional difficulties (Fredriksen et al., 2004; Gregory \& Sadeh, 2012; Pasch et al., 2011; Roberts et al., 2009; Silva et al., 2011; Wolfson \& Carksadon, 1998). To further understand the connection between sleep and academic or psychosocial outcomes, one must determine if a causal link exists, as found in a handful of experimental sleep restriction or extension studies in natural settings (Beebe, Ris, et al., 2008; Beebe, Rose, \& Amin, 2010; Fallone, Acebo, Seifer, \& Carskadon, 2005; Gregory \& Sadeh, 2012; Gruber, Cassoff, Frenette, Wiebe, \& Carrier, 2012; Sadeh, Gruber, \& Raviv, 2003).

\section{Inconsistent Sleep Patterns}

Weekend delay and weekend oversleep served as two indicators of inconsistent sleep patterns (O’Brien \& Mindell, 2005; Wolfson \& Carskadon, 1998). Not surprisingly, the gap between school and non-school night sleep times widened during the second examination. The use of both parental and youth reports of predictors and outcomes adds to the existing literature that suggests that this discrepancy takes its toll on school-related outcomes (O'Brien \& Mindell, 2005; Wolfson \& Carskadon, 1998). For instance, sleeping longer on the weekends (as reported 
by the parents) related to youth reports of more school problems and a negative attitude toward their teachers.

With regard to psychosocial functioning, irregular sleep patterns related to a variety of emotional problems. Consistent with El-Sheikh et al. (2010) who examined a younger sample, inconsistent sleep habits were more apparent among children who were rated by their parents as exhibiting internalizing symptoms, particularly anxiety and somatic complaints. Longer selfreported weekend delay was associated with self-reported anxiety. Additionally, youth who reported that they slept longer on non-school nights than school nights also tended to report feelings of inadequacy and their parents perceived them to have more difficulties with somatic complaints and unusual behaviors and thinking. One interesting finding is that a later parentreported weekend delay at timel uniquely contributed to their reports of their child's tendency to somaticize and youth reports of lower self-esteem, poor adjustment, and emotional difficulties. Given that there were several years in between time1 and time2, it is possible that there is another intervening variable not measured in this study that could explain these relations. For instance, it is possible that youth had more consistent sleep schedules when they were younger because their parents more closely monitored their bedtimes. Parental monitoring may contribute to higher self-perceptions and overall wellbeing (Shek, 2007). In contrast to other studies (O’Brien \& Mendel, 2005; Pasch et al., 2011), we did not find sensation seeking or behavioral difficulties to be uniquely related to inconsistent sleep patterns. However, we did not examine specific risk-taking behaviors such as sexual behaviors and alcohol use.

\section{Daytime Sleepiness}

Consistent with previous research (Dewald et al., 2010), our study found that nearly onethird of youth (33.00\%) reported feeling sleepy during the day. However, only $14.40 \%$ of parents 
indicated that their child evidenced EDS. Thus, parents may not be aware that their children are experiencing sleepiness and it may be that their endorsement reflects the most severe cases. Nonetheless, despite fewer parents reporting this as a problem, parent-reported EDS related to their child's reports of school-related problems. Youth who endorsed daytime sleepiness were more likely to have lower grades.

With regard to psychosocial functioning, self-reported EDS may be indicative of underlying internalizing symptoms such as anxiety. Additionally, children whose parents reported their child exhibited concurrent sleepiness also are more likely to indicate they experienced internalizing symptoms particularly depression, an external locus of control, social stress, feelings of inadequacy, and unusual thoughts and behaviors. Further, the data suggest that parents who report that their child is sleepy during elementary school are at-risk for subsequent behavioral difficulties including both internalizing (e.g., depression) and externalizing problems (e.g., hyperactivity). Thus, it may be important to obtain parents' perceptions of how tired they perceive their child to be when it comes to understanding problematic behaviors exhibited at school. Teachers may also be good sources of information related to daytime sleepiness as they may observe their students yawning, shutting their eyes, putting their heads on their desk, or actually falling asleep in class.

Youth may be sleepy because they are not getting enough sleep or because they are spending less time in deeper stages of sleep (Perfect et al., 2012). Evidence exists that certain nighttime behaviors (e.g., playing video games, drinking caffeine) delay sleep onset and sleep efficiency as well as alter sleep architecture, such as contributing to reduced slow wave sleep (e.g., Dworak, Schierl, Bruns, \& Strüder, 2007; Paterson, Nutt, Ivarsson, Hutson, \& Wilson, 2009). Thus, although few interventions directly target sleepiness, school psychologists may 
facilitate school-wide efforts to increase total sleep time and help youth engage in healthy sleep hygiene practices, which may in turn decrease arousals and increase time spent in deeper sleep.

\section{Insomnia Symptoms}

For this study, if a student or parent endorsed any insomnia symptoms, such as difficulties falling asleep, staying asleep, or early morning awakening (Goodwin et al., 2010), they were considered to have a problem in this area. Using these criteria, over half of the youth endorsed one or more symptoms. Youth with current insomnia symptoms may receive lower grades and/or experience more problems in school. They may also exhibit difficulties with adjustment, mood and behavioral dysregulation, externalizing behaviors (i.e., conduct problems, hyperactivity), internalizing symptoms (i.e., anxiety, depression, somatic complaints), unusual behaviors and thoughts, attention problems, perceived incompetency, and/or have less control over situations, report lower levels of self-esteem or higher levels of stress, or withdrawal from others. Insomnia symptoms relating to both BASC-2 PRF and SRP subscales and composites provides multi-informant support regarding the association of insomnia symptoms and compromised psychosocial functioning. As a result, our recommendation is that in addition to sleep duration and schedules, school psychologists should ask about difficulties with sleep onset and/or maintenance. For youth who are positive in the screening, further evaluation should be conducted to determine if they have insomnia and the extent to which the symptoms impact their learning and/or classroom behaviors.

\section{Sleep-Disordered Breathing}

SDB has been found to be related to inattentiveness (Beebe, Ris, et al., 2010; Mulvaney et al., 2005; Perfect, Archbold, Goodwin, \& Levine-Donnerstein, 2013), aggressivity (Mulvaney et al., 2005; Perfect et al., 2013), oppositionality (Mulvaney et al., 2005), hyperactivity (Beebe, 
Ris, et al., 2010), anxiety (Beebe et al., 2010), depression (Beebe et al., 2010), social problems (Mulvaney et al., 2005; Perfect et al., 2013; Zhao et al., 2008), somatic complaints (Zhao et al., 2008), and poor adaptive functioning (Perfect et al., 2013). In our study, it significantly contributed to poor adaptive behaviors, behavioral problems, depression, and withdrawal. Future research needs to further examine the link between SDB and adaptive behaviors, such as how children get along with others and how they take care of themselves. For instance, when examining whether youth who had remitted, incident (emerged at time2), or persistent (SDB at both timepoints) in this cohort, Perfect et al. (2013) found that youth with current and/or persistent SDB were at-risk for impairment on the Adaptive Behavior Assessment System- $2^{\text {nd }}$ Edition subscales and composites. With regard to the possibility of school psychologists screening for SDB, the only definitive way to confirm SDB is objective testing with PSG or in some cases limited channel home sleep testing; however, surrogates of SDB such as snoring, gasping for air during sleep, and mouth breathing may be useful in identifying children at-risk for SDB and referring them for additional evaluation.

\section{Additional Considerations and Limitations}

With the exception of Letter-Word Identification, the standardized measures of achievement used in this study were primarily fluency. Future studies should include additional measures of academic proficiency, problem-solving ability, and/or advanced knowledge/comprehension to provide more information about the role of sleep in learning and processing. Further, the school performance data were based on parental reports. Obtaining actual grades or performance on state standardized test scores (e.g., Buckhalt et al. 2007; Buckhalt et al. 2009; El-Sheikh et al., 2007; El-Sheikh et al., 2010; Perfect et al., 2012) would provide more objective data and align with Beebe, Ris, et al.'s (2010) contention that indicators 
of day-to-day classroom performance might better reflect learning affected by sleep problems than do laboratory tests.

Although there are currently several questionnaires designed to capture different aspects of sleep, to date, available evaluation methods for many sleep problems such as insomnia and EDS in the pediatric population do not meet the standards for psychometrics (Spruyt \& Gozal, 2011). Further, at the time the study was implemented, there were no standard pediatric sleep questionnaires. Although some were emerging at the time of the second examination, we retained the original questions for consistency. Regarding objective measurements of sleep, a PSG yields some indicators of sleep quality (i.e., sleep efficiency as measured by the PSG); however, we focused on variables that could routinely and efficiently be used as part of a clinical interview during an evaluation in the school setting. In the absence of an actigraphy or sleep diary that takes objective and self-reported sleep timing over multiple days repeatedly, even the most commonly used questionnaires (e.g., School Sleep Habits Survey, Children's Sleep Habit Questionnaire) use similarly worded items for sleep duration asking parents or youth about typical sleep timing. Such research has found that these responses are strongly correlated with objectively-measured sleep using actigraphy (Wolfson et al., 2003). Furthermore, in this study, parents and participants answered questions about the child's sleep and participants underwent an overnight home-based PSG, three hours of neurocognitive testing that included the measures selected for these analyses, and several other procedures. Thus, when screening for sleep problems, having a few targeted questions related to EDS, insomnia symptoms, and sleep duration may be more practical. Additionally, it is possible that school and mental health difficulties precipitate sleep disturbances (Gregory \& Sadeh, 2012) rather than sleep problems 
contributing to poor school and psychosocial outcomes; however, observations by others suggest that adjustment problems follow sleep problems (Fredriksen et al., 2004).

The study has several limitations. First, there was approximately $30 \%$ attrition from time1 to time2, less had all child data (e.g., PSG, BASC-2 SRP), and even fewer of those had the BASC-2 PRF. Although some differences existed with respect to ethnicity, IQ, and age between participants and non-participants, we do not believe they account for our findings, given the consistency across multiple domains. Nonetheless, the results may not apply to Hispanics or those with lower IQs. Second, since the BASC-2 and most of the WJ III ACH subscales (with the exception of the Basic Word Reading subtest) were added during the second examination, it was not possible to ascertain these participants' baseline levels of academic and psychosocial functioning using these measures. Third, with the exception of sleep duration, weekend oversleep, and weekend delay, the sleep parameters were coded as present or absent rather than reflecting the degree of severity or a score on a standardized questionnaire. However, dichotomous classification is a standard method of describing these conditions in the clinical context and has been used in several publications in this cohort (Goodwin et al., 2003; Goodwin et al., 2010; Silva et al., 2011; Zhao et al., 2008). Further, we did not use objective measurements on multiple nights to capture sleep patterns as has been studied by El-Sheikh, Buckhalt and colleagues in elementary school children. Even a diary would have captured self-reported sleep patterns. Although we took a cross-informant approach incorporating both parent- and selfreported predictor and outcome variables, there is the risk of item overlap or bias in some of the models in which a particular sleep problem significantly related to the criterion based on the same informant (Moore et al., 2009). In this regard, obtaining teacher ratings to capture students' classroom behaviors would have enhanced this study (Fallone et al., 2005; Moore et al., 2009). 
Finally, another potential limitation is that sleep studies were conducted on either a school night or non-school night for all participants. Although the literature does indicate differences between sleep patterns on school nights versus non-school nights, we have previously demonstrated that SDB symptoms show little night-to-night variability. Thus, RDI should not have been impacted by when the youth completed the PSG.

\section{Conclusions: Implications for Practice in School Psychology}

Overall, our findings suggest that youth report multiple sleep problems. Parents may underestimate the number of sleep problems experienced by youth, but they are helpful in identifying psychological morbidities, as a result of the sleep problems. When a student is identified as exhibiting learning or behavioral challenges, school psychologists should determine if sleep problems could be underlying these difficulties. They may decide to use currently available questionnaires related to sleep, such as the Sleep Disorders Inventory for Students (Luginbuehl et al., 2008), Children's Sleep Habits Questionnaire (Owens, Spirito, \& McGuinn, 2000), Sleep Self-Report (Owens, Maxim, Nobile, McGuinn, \& Msall, 2000), School Sleep Habits Survey (Wolfson \& Carskadon, 1998; Wolfson et al., 2003) or the Pediatric Sleep Questionnaire (Chervin, 2000; Chervin, Hedger, Dillon, \& Pituch, 2000; for a review of these and other measures, see Lewandowski, Toliver-Sokol, \& Palmero, 2011). However, the limited psychometric properties of many of these questionnaires should be taken into account, particularly for assessing the range of sleep problems and not just sleep disorders. Alternatively, a school psychologist may choose to use a series of questions as suggested in this article, as part of a semi-structured interview. One helpful framework is the "BEARS," which stands for Bedtime problems, Excessive daytime sleepiness, Awakenings during the night, Regularity and duration of sleep, and Snoring. It suggests a set of screening questions based on the student's 
age. It is currently available at

http://books.google.com/books?id=7LBGG0ht4qMC\&pg=PA10\&lpg=PA10\&dq=BEARS+sleep +clinical+interview\&source=bl\&ots=zAhsc_rQa3\&sig=GUgk7MEyF2_0BphhtKJmIvYdhpE\&h

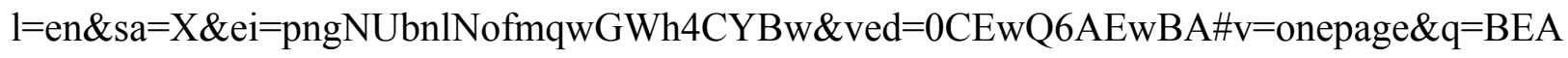
RS\%20sleep\%20clinical\%20interview\&f=false or can be found in Mindell and Owens (2003. In particular, a school psychologist screening for sleep problems may find that daytime sleepiness and insomnia symptoms are related to problems in school and mental health difficulties (Roberts et al., 2002, 2008, 2009). If it is determined that a child is not sleeping long enough, has irregular sleep patterns, is very tired, has difficulties with sleep onset or maintenance, or is at-risk for $\mathrm{SDB}$, school professionals may wish to discuss the role of sleep with parents and make a plan for targeting these problems or making appropriate referrals. In the absence of sleep interventions, children may continue to struggle with overall school performance and academic success, in addition to exhibiting challenging classroom behaviors, despite school-based interventions and/or accommodations. 


\section{References}

Beebe, D.W., Fallone, G., Godiwala, N., Flanigan, M., Martin, D., Schaffner, L., \& Amin R. (2008). Feasibility and behavioral effects of an at-home multi-night sleep restriction protocol for adolescents. Journal of Child Psychology and Psychiatry, 49, 915-923.

Beebe, D. W., Ris, M. D., Kramer, M. E., Long, E., \& Amin, R. (2010). The association between sleep disordered breathing, academic grades, and cognitive and behavioral functioning among overweight subjects during middle to late childhood. Sleep, 33, 144756.

Beebe, D.W, Rose, D., \& Amin, R. (2010). Brief report: performance of experimentally sleeprestricted adolescents in a simulated classroom. Journal of Adolescent Health, 47, 523-525.

Bub, K. L., Buckhalt, J.A. \& El-Sheikh, M. (2011). Children's sleep and cognitive performance: A cross-domain analysis of change over time. Developmental Psychology, 47, 1504-14. doi:10.1037/a0025535

Buckhalt, J.A., El-Sheikh, M., \& Keller. P. (2007). Children's sleep and cognitive functioning: Race and socioeconomic status as moderators of effects. Child Development, 78, 213 231.

Buckhalt, J.A., El-Sheikh, M., Keller. P., \& Kelley, R. (2009). Concurrent and longitudinal relations between children's sleep and cognitive functioning. Child Development, 80, 875-892.

Buckhalt, J. A., Wolfson, A. R., \& El-Sheikh, M. (2009). Children's sleep and school psychology practice. School Psychology Quarterly, 24, 60-69. doi:10.1037/a0014497

Centers for Disease Control. Body Mass Index. http://www.cdc.gov/healthyweight/assessing/bmi/ Last accessed June 29, 2012. 
Chervin, R.D. (2000). Pediatric Sleep Questionnaire (PSQ): Validity \& reliability of scales for sleep disordered breathing, snoring, sleepiness, and behavioral problems. Sleep Medicine, 1, 21-32.

Chervin, R.D., Hedger, K., Dillon, J.E., \& Pituch, K.J. (2000). Pediatric Sleep Questionnaire (PSQ): Validity and reliability of scales for sleep-disordered breathing, snoring, sleepiness, and behavioral problems. Sleep Medicine Reviews, 1, 21-32.

Chung, K., Kan, K. K., \& Yeung, W. (2011). Assessing insomnia in adolescents: Comparison of insomnia severity index, Athens insomnia scale and sleep quality index. Sleep Medicine, 12, 463-70. doi:10.1016/j.sleep.2010.09.019

Cohen, J. (1988). Statistical power analysis for the behavioral sciences (2nd ed.). New Jersey: Lawrence Erlbaum.

Dahl, R.E. (1995). Sleep in behavioral and emotional disorders. In R. Ferber \& M.H. Kryger (Eds.), Principles and Practice of Sleep Medicine in the Child (pp. 148-149). Philadelphia: W.B. Saunders.

Dewald, J.F., Meijer, A.M., Oort, F.J., Kerkof, G. A., \& Bogels, S.M. (2010). The influence of sleep quality, sleep duration and sleepiness on school performance in children and adolescents: A meta-analytic review. Sleep Medicine Reviews, 14, 179-189. doi:10.1016/j.smrv.2009.10.004

Dworak, M., Schierl, T., Bruns, T., \& Strüder, H.K. (2007). Impact of singular excessive computer game and television exposure on sleep patterns and memory performance of school-aged children. Pediatrics, 120, 978-985.

El-Sheikh, M., Buckhalt, J.A., Keller, P.S., Cummings, E.M., \& Acebo,C. (2007). Child emotional security and academic achievement: The role of sleep disruptions. Journal of 
Family Psychology, 21, 29-38.

El-Sheikh, M., Kelly, R.J., Buckhalt, J.A. \& Hinnant, J.B. (2010). Children's sleep and adjustment over time: The role of socioeconomic context. Child Development, 81, 870-883.

Fallone G., Acebo, C., Seifer, R., \& Carskadon, M.A. (2005). Experimental restriction of sleep opportunity in children: Effects on teacher ratings. Sleep, 28, 1561-1567.

Fredericksen, K., Reddy, R., Rhodes, J. \& Way, N. (2004). Sleepless in Chicago: The influence of sleep patterns on the psychological adjustment of middle school students. Child Development,75, 84-95.

Gaultney, J.F. (2011). The prevalence of sleep disorders in college students: Impact on academic performance. Journal of the American College of Health, 59, 91 - 97.

Gibson, E. S., Powles, A.C P., Thabane, L., O’Brien, S., Molnar, D.S., Trajanovic, N., . . . Chilcott-Tanser, L. (2006). "Sleepiness" is serious in adolescence: Two surveys of 3235 Canadian students. BMC Public Health, 6, 116. doi:10.1186/1471-2458-6-116

Gomez, R.L., Newman-Smith, K.C., Breslin, J.H., \& Bootzin, R.R. (2010). Learning, memory and sleep in children. Journal of Clinical Sleep Medicine, 6, 45-57. doi:10.1016/j.jsmc.2010.12.002

Goodwin, J., Enright, P., Kaemingk, K., Rosen, G., Morgan, W., Fregosi, R.F., \& Quan, S.F. (2001). Feasibility of using unattended polysomnography in children for research: Report of the Tucson Children's Assessment of Sleep Apnea study (TuCASA). Sleep, 24, 937-944.

Goodwin, J., Kaemingk, K., Fregosi, R.F., Rosen, G., Morgan, W., . . . \& Quan, S.F. (2003). Clinical outcomes associated with sleep disordered breathing in Caucasian and Hispanic children - the Tucson Children's Assessment of Sleep Apnea Study (TuCASA). Sleep, 26, 587-591. 
Goodwin, J.L., Vasquez, M.M., Silva, G.E., \& Quan, S.F. (2010). Incidence and remission of sleep-disordered breathing and related symptoms in 6- to 17- year old children - The Tucson Children's Assessment of Sleep Apnea Study. The Journal of Pediatrics, 157, 5761. doi:10.1016/j.jpeds.2010.01.033

Gregory, A.M., \& Sadeh, A. (2012). Sleep, emotional and behavioral difficulties in children and adolescents. Sleep Medicine Reviews, 16, 129-36.

Gruber, R., Cassoff, J., Frenette, S., Wiebe, S., \& Carrier, J. (2012). Impact of sleep extension and restriction on children's emotional lability and impulsivity. Pediatrics, 13, e1155-e1161.

Joo, S., Shin, C., Kim, J., Yi, H., Ahn, Y., Park, M. . . . Lee, S. (2005) Prevalence and correlates of excessive daytime sleepiness in high school students in Korea. Psychiatry and Clinical Neurosciences, 59, 433-40.

Kaemingk, K.L., Pasvogel, A.E., Goodwin, J.L., Mulvaney, S. A., Martinez, F. Enright, P.L., . . . Quan, S.F. (2003). Learning in children and sleep disordered breathing: Findings of the Tucson Children's Assessment of Sleep Apnea (TuCASA) Prospective Cohort Study. Journal of the International Neuropsychological Society, 9, 1016-26. doi:10.1017/S1355617703970056

Kahn A, Van de Merckt C, Rebuffat E, Mozin, M.J., Sottiaiux, M., ... Hennart, P. (1989). Sleep problems in healthy preadolescents. Pediatrics, 84, 542-546.

Lazaratou, H., Dikeos, D. G., Anagnostopoulos, D. C., Sbokou, O. \& Soldatos, C. R. (2005). Sleep problems in adolescence: A study of senior high school students in Greece. European Child \& Adolescent Psychiatry, 14, 237-43. doi:10.1007/s00787-005-0460-0

Lewandowski, A.S., Toliver-Sokel, M., \& Palermo, T.M. (2011). Evidence-based review of subjective pediatric sleep measures. Journal of Pediatric Psychology, 36, 780-793. 
doi:10.1093/jpepsy/jsq119

Luginbuehl, M., Bradley-Klug, K. L., Ferron, J., Anderson, W. M., \& Benbadis, S. R. (2008).

Pediatric sleep disorders: Validation of the sleep disorders inventory for students. School Psychology Review, 37, 409-431.

Mayes, S.D., Calhoun, S.L., Bixler, E.O., \& Vgontzas, A.N. (2008). Nonsignificance of sleep relative to IQ and neuropsychological scores in predicting academic achievement. Journal of Developmental \& Behavioral Pediatrics, 29, 206-212.

Mindell, J. A., Emslie, G., Blumer, J., Genel, M., Glaze, D., Ivanenko, A., . . .Banas, B. (2006). Pharmacological management of insomnia in children and adolescents: Consensus statement. Pediatrics, 117, e1223-32. doi:10.1542/peds.2005-1693

Mindell, J.A., \& Owens, J. A. (2003). Clinical Guide to Pediatric Sleep: Diagnosis and Management of Sleep Problems. Philadelphia: Lippincott Williams \& Wilkins.

Moore, H., Kirchner, H.L., Drotar, D., Johnson, N., Rosen, C., Ancoli-Israel S., \& Redline, S. (2009). Relationships among sleepiness, sleep time, and psychological functioning in adolescents. Journal of Pediatric Psychology, 34, 1175-1183.

Mulvaney, S.A., Goodwin, J.L., Morgan, W.J., Rosen, G.R., Quan, S.F., \& Kaemingk, K.L. (2005). Behavior problems associated with sleep disordered breathing in school-aged children - the Tucson Children's Assessment of Sleep Apnea Study. Journal of Pediatric Psychology, 31, 322-30. doi:10.1093/jpepsy/jsj035

Nagelkerke, N. J. D. (1991). A note on a general definition of the coefficient of determination. Biometrika, 78, 691-692.

O’Brien, E.M., \& Mindell, J.A. (2005). Sleep and risk-taking behavior in adolescents. Behavioral Sleep Medicine, 3, 113-133. 
O’Brien, L. M., Mervis, C. B., Holbrook, C. R., Bruner, J. L., Smith, N. H., McNally, N. . . Gozal, D. (2004). Neurobehavioral correlates of sleep-disordered breathing in children. Journal of Sleep Research, 13, 165-172.Owens, J.A., Maxim, R., Nobile, C,, McGuinn, M,, \& Msall, M. (2000). Parental and self-report of sleep in children with attentiondeficit/hyperactivity disorder. Archive of Pediatric and Adolescent Medicine, 154, 549-555.

Owens, J. A., Spirito, A., \& McGuinn, M. (2000). The children's sleep habits questionnaire (CSHQ): Psychometric properties of a survey instrument for school-aged children. SLEEP, 23, 1043-1051.

Pasch, K.E., Nelson, M.C., Lytle, L.A., \& Moe, S.G. (2010). Adolescent sleep, risk behaviors, and depressive symptoms: Are they linked? American Journal of Health Behavior, 34, 237-48.

Pagel, J.F., Forister, N., \& Kwiatkowski, C. (2007). Adolescent sleep disturbance and school performance: The confounding variable of socioeconomics. Journal of Clinical Sleep Medicine, 3, 19-23.

Pagel, J. F., \& Kwiatkowski, C. F. (2010). Sleep complaints affecting school performance at different educational levels. Frontiers in Neurology, 1, 1-6.doi:10.3389/fneur.2010.00125

Paterson, L.M., Nutt, D.J., Ivarsson, M., Hutson, P.H., \& Wilson SJ. (2009). Effects on sleep stages and microarchitecture of caffeine and its combination with zolpidem or trazodone in healthy volunteers. Journal of Psychopharmacology, 23, 487-494.

Perez-Chada, D., Perez-Lloret, S., Videla, A. J., Cardinali, D., Bergna, M. A., FernandezAcquier, M., ... Drake, C. (2007). Sleep disordered breathing and daytime sleepiness are associated with poor academic performance in teenagers. A study using the Pediatric Daytime Sleepiness Scale (PDSS). Sleep, 30, 1698-1703. 
Perfect, M.M., Archbold, K., Goodwin, J., Levine-Donnerstein, D., \& Quan, S.F. (2013). Risk of difficulties in behavioral and adaptive functioning in youth with persistent and current sleep-disordered breathing. SLEEP, 26, 517 - 525.

Perfect, M.M., Patel, P.G., Scott, R.E., Wheeler, M.D., Patel, C., . . Quan, S.F. (2012). Sleep, glucose, and daytime functioning in youth with type 1 diabetes. SLEEP, 35, 81 - 88.

Rechtschaffen A., \& Kales A.A. (1968). Manual of Standardized Terminology: Techniques and Scoring Systems for Sleep Stages of Human Subjects. Los Angeles: UCLA Brain Information Service/Brain Research Institute.

Reynolds, C. R., \& Kamphaus, R. W. (2004). Behavior Assessment System for Children Second Edition manual. Circle Pines, MN: American Guidance Service Publishing.

Roberts, R.E., Roberts, C.R., \& Chen, I.G. (2002). Impact of insomnia on future functioning of adolescents. Journal of Psychosomatic Research, 53, 561-569.

Roberts, R.E., Roberts, C.R., \& Duong, H.T. (2008). Chronic insomnia and its negative consequences for health and functioning of adolescents: A 12-month prospective study. Journal of Adolescent Health, 42, 294-302. ‘

Roberts, E., Roberts, C. \& Duong, H.T. (2009). Sleepless in adolescence: Prospective data on sleep deprivation, health and functioning. Journal of Adolescence, 32, 1045-1057. PMID: 19361854

Sadeh, A., Gruber, R., \& Raviv, A. (2003). The effects of sleep restriction and extension on school-age children: What a difference an hour makes. Child Development, 7, 444-455.

Shin, C., Kim, J., Lee, S., Ahn, Y. \& Joo, S. (2003). Sleep habits, excessive daytime sleepiness and school performance in high school students. Psychiatry and Clinical Neurosciences, 57, 451-453. 
Short, M.A., Gradisar, M., Wright, H., Lack, L.C., Dohnt, H., \& Carskadon, M.A. (2011). Time for bed: Parent-set bedtimes associated with improved sleep and daytime functioning of adolescents. Sleep, 34, $797-800$.

Silva, G.E., Goodwin, J.L., Parthasarathy, S., Sherrill, D.L., Vana, K.D., Drescher, A.A., Quan, S.F. (2011). Longitudinal association between short sleep, body weight, and emotional and learning problems in Hispanic and Caucasian children. Sleep, 34, $1197-$ 1205. doi: 10.5665/SLEEP.1238

Spruyt, K., \& Gozal, D. (2011). Pediatric Sleep Questionnaires as diagnostic or epidemiological tools: A review of currently available instruments. Sleep Medicine Reviews, 15, 19-32.

Walker, M.P. (2009). The role of sleep in cognition and emotion. Annals of the New York Academy of Sciences. 1156, 181-183.

Wechsler, D. (1999). Wechsler Abbreviated Scale of Intelligence WASI: manual. San Antonio, TX: Pearson/PsychCorp.

Wolfson, A. R., \& Carskadon, M. A. (1998). Sleep schedules and daytime functioning in adolescents. Child Development, 69, 875-887.

Wolfson, A.R., Carskadon, M.A., Acebo, C., Seifer, R., Fallone, G., Labyak, S.E.,\& Martin, J.L. (2003). Validation of a sleep habits survey for adolescents. Sleep, 26, 213-216.

Woodcock, R. W., McGrew, K. S., \& Mather, N. (2001a). Woodcock-Johnson III Tests of Achievement. Itasca, IL: Riverside Publishing.

Yoo, S.S., Gujar N., Hu P., Jolesz, F.A. \& Walker, M.P. (2007). The human emotional brain without sleep: A prefrontal-amygdala disconnect. Current Biology, 17, 877-878 
Zhao, Q., Sherrill, D. L., Goodwin, J. L., \& Quan, S. F. (2008). Association between sleep disordered breathing and behavior in school-age children: The Tucson Children's Assessment of Sleep Apnea study. Open Epidemiological Journal, 1, 1-9. 
Table 1

Sample Characteristics and Sleep Problems of Participants who Had Complete Child Data at the Second Examination

\begin{tabular}{|c|c|c|c|}
\hline \multirow{2}{*}{ Characteristic } & \multicolumn{3}{|c|}{ Parent Report (time2) } \\
\hline & Parent Report (time1) & & Youth Report (time2) \\
\hline Age (years) & $\begin{array}{c}8.69+/-1.60(6.0- \\
12.3)\end{array}$ & $\begin{array}{c}13.24+/-1.69(9.93- \\
17.87)\end{array}$ & \\
\hline \multicolumn{4}{|l|}{ Gender } \\
\hline Male & $50.8 \%$ & & \\
\hline Female & $49.2 \%$ & & \\
\hline \multicolumn{4}{|l|}{ Ethnicity } \\
\hline Hispanic or Latino & $68.2 \%$ & & \\
\hline Not Hispanic or Latino & $31.8 \%$ & & \\
\hline Body Mass Index (BMI; z-score) & $\begin{array}{c}.30+/-1.33(-6.96- \\
3.32)\end{array}$ & $.43+/-1.18(-3.81-2.78)$ & \\
\hline Sleep Duration (week; hours) & $\begin{array}{c}9.68+/-.72(7.50- \\
11.50)\end{array}$ & $9.13+/-.97(6.00-12.50)$ & $\begin{array}{c}8.92+/-1.23(4.88- \\
12.63)\end{array}$ \\
\hline Sleep Duration (non-school nights) & $\begin{array}{c}9.69+/-.94(7.00- \\
12.50\end{array}$ & $\begin{array}{c}9.56+/-1.29(5.00- \\
14.00)\end{array}$ & $\begin{array}{c}9.28+/-1.75(3.00- \\
13.25)\end{array}$ \\
\hline Sleep Duration (school nights) & $\begin{array}{c}9.67+/-.78(7.00- \\
12)\end{array}$ & $\begin{array}{c}8.70+/-1.00(5.00- \\
11.50)\end{array}$ & $\begin{array}{c}8.56+/-1.32(3.00- \\
12.00)\end{array}$ \\
\hline Weekend Oversleep & $\begin{array}{c}.01+/-1.26(-2.5- \\
3.0)\end{array}$ & $.86+/-1.26(-3.00-4.50)$ & $.71+/-1.89(-7.00-8.00)$ \\
\hline Weekend Delay & $\begin{array}{c}.94+/-.62(-1.00- \\
3.00)\end{array}$ & $1.22+.91(-.50-5.50)$ & $1.51+/-1.22(-1.50-6.00)$ \\
\hline Excessive Daytime Sleepiness (\%) & $14.40 \%$ & $14.40 \%$ & $31.10 \%$ \\
\hline Insomnia Symptoms (\%) & $25.00 \%$ & $24.00 \%$ & $51.50 \%$ \\
\hline Sleep-Disordered Breathing ${ }^{\mathrm{a}}(\%)$ & $26.50 \%$ & $16.70 \%$ & \\
\hline
\end{tabular}

Note: Means +/- standard deviations are reported; ranges are in parentheses. ${ }^{a}$ Based on polysomnography recordings. 
Table 2.

Means Scores, Standard Deviations, and Ranges on the Behavior Assessment Scale for Children (BASC)-Parent Report

\begin{tabular}{|c|c|c|c|}
\hline Scale Name & Mean & SD & Range \\
\hline $\begin{array}{l}\text { Behavioral Symptoms } \\
\text { Index }^{\mathrm{a}}\end{array}$ & 48.87 & 9.59 & $34-84$ \\
\hline $\begin{array}{l}\text { Adaptive Behavior } \\
\text { Composite }^{\text {b }}\end{array}$ & 50.07 & 10.01 & $24-69$ \\
\hline Social Skills & 49.02 & 10.05 & $23-69$ \\
\hline Leadership & 51.41 & 10.05 & $34-89$ \\
\hline $\begin{array}{l}\text { Externalizing } \\
\text { Composite }^{\mathrm{a}}\end{array}$ & 49.00 & 9.30 & $34-90$ \\
\hline Hyperactivity & 49.27 & 10.15 & $34-89$ \\
\hline Aggression & 48.65 & 8.13 & $37-82$ \\
\hline Conduct Problems & 49.08 & 8.94 & $37-87$ \\
\hline $\begin{array}{l}\text { Internalizing } \\
\text { Composite }^{\mathrm{a}}\end{array}$ & 48.28 & 9.91 & $31-85$ \\
\hline Anxiety & 48.54 & 11.17 & $30-96$ \\
\hline Depression & 49.43 & 9.74 & $37-86$ \\
\hline Somatization & 48.31 & 8.51 & $36-90$ \\
\hline Atypicality & 48.53 & 8.510 & $41-80$ \\
\hline Withdrawal & 49.17 & 9.56 & $35-83$ \\
\hline Attention Problems & 49.44 & 9.81 & $35-76$ \\
\hline
\end{tabular}

Note: ${ }^{\mathrm{a}}=$ Higher scores reflect more problem on these Composites and/or subscales; ${ }^{\mathrm{b}}=$ Lower scores reflect more problems on these composites and/or subscales 
Table 3.

Means and standard deviations of the scores on the Behavior Assessment Scale for Children-Self Report-2 ${ }^{\text {nd }}$ Edition (BASC-2) based on sleep-disordered breathing (SDB) history

\begin{tabular}{|c|c|c|c|}
\hline Scale Name & Mean & SD & Range \\
\hline Emotional Symptoms Index ${ }^{a}$ & 46.99 & 9.21 & $28-86$ \\
\hline Personal Adjustment Composite & 9.05 & 52.27 & $26-76$ \\
\hline Relations with Parents & 50.27 & 10.28 & $21-67$ \\
\hline Interpersonal Relations & 53.32 & 7.69 & $26-62$ \\
\hline Self-Esteem & 52.76 & 8.14 & $20-62$ \\
\hline Self-Reliance & 52.16 & 8.41 & $23-71$ \\
\hline BASC School Problems Composite ${ }^{a}$ & 50.88 & 10.96 & $27-84$ \\
\hline Attitude to School & 49.82 & 10.71 & $32-78$ \\
\hline Attitude to Teachers & 50.09 & 10.97 & $34-86$ \\
\hline Sensation Seeking (adolescent only) & 52.28 & 10.81 & $26-74$ \\
\hline Internalizing Composite $^{\mathrm{a}}$ & 47.29 & 8.64 & $34-86$ \\
\hline Depression & 46.46 & 8.31 & $40-87$ \\
\hline Anxiety & 48.00 & 9.28 & $32-80$ \\
\hline Sense of Inadequacy & 47.66 & 9.60 & $35-96$ \\
\hline Somatization & 48.02 & 8.45 & $34-77$ \\
\hline Inattention/Hyperactivity $^{\mathbf{a}}$ & 49.54 & 10.88 & $31-83$ \\
\hline Attention Problems & 49.32 & 10.75 & $34-77$ \\
\hline Hyperactivity & 49.90 & 10.74 & $33-90$ \\
\hline \multicolumn{4}{|l|}{ Additional Subscales $^{\mathrm{a}}$} \\
\hline Atypicality & 48.18 & 8.35 & $38-79$ \\
\hline Locus of Control & 49.84 & 9.66 & $36-80$ \\
\hline Social Stress & 46.57 & 8.82 & $34-80$ \\
\hline
\end{tabular}


Table 4

Hierarchical Regression Results Predicting the Behavior Assessment Scale (BASC-2) for Children-Parent Report Form (PRF) Behavioral Symptoms Index (BSI), the BASC-Self Report of Personality Form (SRP) Emotional Symptoms Index, and the BASC-PRF Internalizing Composite

\begin{tabular}{|c|c|c|c|c|c|c|}
\hline \multirow{2}{*}{ Model } & PRF & $\mathrm{BSI}^{\mathrm{b}}$ & SRP & $\mathrm{ESI}^{\mathrm{c}}$ & PRF & Internalizing $^{\mathrm{d}}$ \\
\hline & $\Delta \mathrm{R}^{2}$ & $\beta$ & $\Delta \mathrm{R}^{2}$ & $\mathrm{~B}$ & $\Delta \mathrm{R}^{2}$ & $\beta$ \\
\hline Step 1 & $.10^{* *}$ & & $.12 * * *$ & & $.10^{* *}$ & \\
\hline \multicolumn{7}{|l|}{ Sociodemographic variables ${ }^{\mathrm{a}}$} \\
\hline Step 2 & $.16^{* *}$ & & $.17 * * *$ & & $.17 * * *$ & \\
\hline Parent-Reported Insomnia Symptoms (time1) & & .07 & & -.04 & & .06 \\
\hline Parent-Reported Insomnia Symptoms (time2) & & $.16^{*}$ & & .03 & & $.18^{*}$ \\
\hline Parent-Reported Daytime Sleepiness (time1) & & $.15^{*}$ & & -.04 & & $.20 * *$ \\
\hline Parent-Reported Daytime Sleepiness (time2) & & .09 & & .09 & & .03 \\
\hline Parent-Reported Sleep Duration (time1) & & .04 & & -.09 & & .09 \\
\hline Parent-Reported Sleep Duration (time2) & & .09 & & .07 & & .09 \\
\hline Parent-Reported Weekend Delay (time1) & & -.01 & & $.17 *$ & & .00 \\
\hline Parent-Reported Weekend Delay (time2) & & .07 & & .04 & & .09 \\
\hline Parent-Reported Weekend Oversleep (time1) & & -.09 & & -.03 & & $-.13+$ \\
\hline Parent-Reported Weekend Oversleep (time2) & & -.00 & & .03 & & .06 \\
\hline Self-Reported Insomnia Symptoms & & $.16^{*}$ & & $.26 * * *$ & & .07 \\
\hline Self-Reported Daytime Sleepiness & & .01 & & .09 & & .04 \\
\hline Self-Reported Sleep Duration & & -.09 & & $-.16^{*}$ & & -.11 \\
\hline Self-Reported Weekend Delay & & -.06 & & .02 & & -.11 \\
\hline Self-Reported Weekend Oversleep & & -.08 & & .03 & & $-.15+$ \\
\hline Sleep-Disordered Breathing (time1) & & -.01 & & -.04 & & $-.13+$ \\
\hline Sleep-Disordered Breathing (time2) & & $.15^{*}$ & & .00 & & $.12+$ \\
\hline Total $\mathrm{R}^{2}$ & & $.26 * * *$ & & $.29 * * *$ & & $.27 * * *$ \\
\hline
\end{tabular}

Note. ${ }^{a}$ Sociodemographic variables included: sex, age, race/ethnicity (Hispanic or non-Hispanic), body mass index, parent's highest education level, income, Verbal IQ, Performance IQ; ${ }^{\mathrm{b}} F(25,217)=2.70, p<.001, R^{2}=.26 ;{ }^{\mathrm{c}} F(25$, $251)=3.67, p<.001, R^{2}=.29 ;{ }^{\mathrm{d}} F(25,217)=2.95, p<.001, R^{2}=.27 ;+p \leq .10 ;{ }^{*} p \leq .05,{ }^{* *} p \leq .01,{ }^{* * *} p \leq .001$ 
Table 5

Hierarchical Regression Results Predicting the Behavior Assessment Scale (BASC) for Children-Self-Report of Personality Form (SRP) Internalizing Composite, the BASC-2 Parent Report Form (PRF), Depression Subscale, and the BASC-2 SRP Depression Subscale

\begin{tabular}{lcccccccc}
\hline & SRP & Internalizing & PRF & & SRP & Depression \\
Model & $\Delta \mathrm{R}^{2}$ & $\mathrm{~B}$ & $\Delta \mathrm{R}^{2}$ & Depression & $\Delta \mathrm{R}^{2}$ & $\beta$ \\
& & & & & $\beta$ & & \\
& & & & & & & \\
\hline
\end{tabular}

Step 1

$.11^{* * *}$

$.08^{*}$

$.08^{* *}$

Sociodemographic variables ${ }^{\mathrm{a}}$

Step 2

$.20 * * *$

$.19^{* * *}$

$.17 * * *$

Parent-Reported Insomnia Symptoms (time1)

$-.05$

.07

$-.01$

Parent-Reported Insomnia Symptoms (time2)

.07

$.12+$

.05

Parent-Reported Daytime Sleepiness (time1)

.03

$.21^{* *}$

.03

Parent-Reported Daytime Sleepiness (time2)

$.14^{*}$

$.13+$

$.14 *$

Parent-Reported Sleep Duration (time1)

$-.04$

.05

$-.06$

Parent-Reported Sleep Duration (time2)

.05

.09

.04

Parent-Reported Weekend Delay (time1)

.06

$-.01$

.02

Parent-Reported Weekend Delay (time2)

.11

.05

$.15+$

Parent-Reported Weekend Oversleep (time1)

$-.03$

$-.13+$

$-.07$

Parent-Reported Weekend Oversleep (time2)

.07

.01

$.15+$

Self-Reported Insomnia Symptoms

$.26 * * *$

$.13^{*}$

$.13^{*}$

Self-Reported Daytime Sleepiness

$.14 *$

.02

.10

Self-Reported Sleep Duration

$-.12+$

$-.09$

$-.18^{*}$

Self-Reported Weekend Delay

$-.03$

$-.03$

$-.06$

Self-Reported Weekend Oversleep

$-.01$

$-.12$

$-.02$

Sleep-Disordered Breathing (time1)

$-.00$

$-.05$

$-.01$

Sleep-Disordered Breathing (time2)

$-.02$

$.17^{*}$

$-.04$

Total $\mathrm{R}^{2}$

$.31 * * *$

$.27 * * *$

Note. ${ }^{a}$ Sociodemographic variables included: sex, age, race/ethnicity (Hispanic or non-Hispanic), body mass index, parent's highest education level, income, Verbal IQ, Performance IQ ${ }^{b} F(25,251)=4.04, p<.001, R^{2}=.31 ;{ }^{\mathrm{c}} F(25$, 217) $=2.83, p<.001, R^{2}=.27 ;{ }^{\mathrm{d}} F(25,251)=2.98, p<.001, R^{2}=.25 ;+p \leq .10,{ }^{*} p \leq .05,{ }^{* *} p \leq .01,{ }^{* * *} p \leq .001$ 
Table 6

Hierarchical Regression Results Predicting the Behavior Assessment Scale (BASC-2) for Children-Self-Report of Personality Form (SRP) Anxiety subscale, the BASC-2 PRF Atypicality Subscale, and the BASC-2 SRP Self-Esteem Subscale

SRP Anxiety PRF Atypicality SRP $\quad$ Personal

Model

$\mathrm{B}$

Adjustment

\begin{tabular}{llllll} 
& $\Delta \mathrm{R}^{2}$ & $\mathrm{~B}$ & $\Delta \mathrm{R}^{2}$ & $\Delta \mathrm{R}^{2}$ & $\mathrm{~B}$ \\
\hline Step 1 & $.07^{*}$ & & $.09^{* *}$ & $.09^{* *}$
\end{tabular}

Sociodemographic variables ${ }^{\mathrm{a}}$

Step 2

$.20^{* * *}$

$.13^{* *}$

$.13^{* *}$

Parent-Reported Insomnia Symptoms (time1)

$-.07$

Parent-Reported Insomnia Symptoms (time2)

.09

.06

.06

Parent-Reported Daytime Sleepiness (time1)

.04

$-.01$

$-.01$

Parent-Reported Daytime Sleepiness (time2)

.05

.08

.08

Parent-Reported Sleep Duration (time1)

$-.04$

$-.09$

$-.09$

Parent-Reported Sleep Duration (time2)

.06 .

.09

.09

Parent-Reported Weekend Delay (time1)

. .06

$-.01$

$-.01$

Parent-Reported Weekend Delay (time2)

$-.07$

$-.17^{*}$

$-.17 *$

Parent-Reported Weekend Oversleep (time1)

$-.00$

.03

.03

Parent-Reported Weekend Oversleep (time2)

.04

$-.01$

$-.01$

Self-Reported Insomnia Symptoms

$-.00$

$-.00$

$.26 * * *$

$-.23^{* * *}$

Self-Reported Daytime Sleepiness

$-.21 * *$

$-.03$

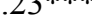

Self-Reported Sleep Duration

$-.15^{*}$

.09

$-.03$

Self-Reported Weekend Delay

$--.15^{*}$

$-.09$

.09

Self-Reported Weekend Oversleep

$--.02$

$-.04$

$-.09$

Sleep-Disordered Breathing (time1)

$--.06$

.01

$-.04$

Sleep-Disordered Breathing (time2)

$-.03$

.03

.01

$.26^{* * *}$

$.22 * * *$

.03

Total $\mathrm{R}^{2}$

.

$.22 * * *$

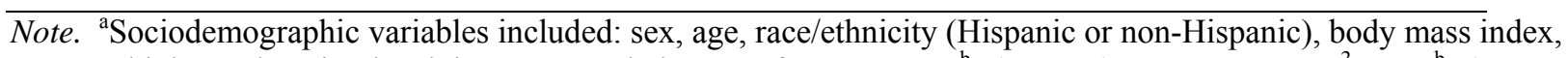
parent's highest education level, income, Verbal IQ, Performance IQ; ${ }^{b} F(25,251)=3.31, p<.001, R^{2}=.27$; ${ }^{\mathrm{b}} F(25$, $217)=2.52, p<.001, R^{2}=.25 ;{ }^{\mathrm{d}} F(25,251)=2.48, p<.001, R^{2}=.22 ;+p \leq .10 ;{ }^{*} p \leq .05,{ }^{* *} p \leq .01,{ }^{* * *} p \leq .001$ 
Table 7

Hierarchical Regression Results Predicting the Behavior Assessment Scale (BASC-2) for Children-Parent Report Form (PRF) Somatization Subscale, the BASC-2 PRF, and the BASC-2 SRP Self-Esteem Subscale

\begin{tabular}{|c|c|c|c|c|c|c|}
\hline \multirow{3}{*}{ Model } & PRF & Somatization & SRP & Locus of & SRP & Self- \\
\hline & \multirow{2}{*}{\multicolumn{2}{|c|}{$\Delta \mathrm{R}^{2}$}} & \multicolumn{3}{|c|}{ Control } & Esteem \\
\hline & & & $\Delta \mathrm{R}^{2}$ & B & $\Delta \mathrm{R}^{2}$ & $\beta$ \\
\hline Step 1 & $.09^{* *}$ & & .04 & & $.09 * *$ & \\
\hline \multicolumn{7}{|l|}{ Sociodemographic variables ${ }^{\mathrm{a}}$} \\
\hline Step 2 & $.14^{* *}$ & & $.15 * * *$ & & $.14 * *$ & \\
\hline Parent-Reported Insomnia Symptoms (time1) & & .10 & & -.05 & & .10 \\
\hline Parent-Reported Insomnia Symptoms (time2) & & -.05 & & .06 & & -.05 \\
\hline Parent-Reported Daytime Sleepiness (time1) & & .02 & & .01 & & .02 \\
\hline Parent-Reported Daytime Sleepiness (time2) & & $-.11+$ & & $.14 *$ & & $-.11+$ \\
\hline Parent-Reported Sleep Duration (time1) & & $.13+$ & & -.08 & & $.13+$ \\
\hline Parent-Reported Sleep Duration (time2) & & -.08 & & .03 & & -.08 \\
\hline Parent-Reported Weekend Delay (time1) & & $-.16^{*}$ & & .04 & & $-.16^{*}$ \\
\hline Parent-Reported Weekend Delay (time2) & & -.09 & & .13 & & -.09 \\
\hline Parent-Reported Weekend Oversleep (time1) & & -.04 & & .02 & & -.04 \\
\hline Parent-Reported Weekend Oversleep (time2) & & .01 & & .02 & & .01 \\
\hline Self-Reported Insomnia Symptoms & & $-.16^{*}$ & & $.26 * * *$ & & $-.16^{*}$ \\
\hline Self-Reported Daytime Sleepiness & & .08 & & .03 & & .08 \\
\hline Self-Reported Sleep Duration & & $.12+$ & & -.09 & & $.12+$ \\
\hline Self-Reported Weekend Delay & & -.01 & & -.02 & & -.01 \\
\hline Self-Reported Weekend Oversleep & & .00 & & .01 & & .00 \\
\hline Sleep-Disordered Breathing (time1) & & -.06 & & .07 & & -.06 \\
\hline Sleep-Disordered Breathing (time2) & & .08 & & .00 & & .08 \\
\hline Total $\mathrm{R}^{2}$ & $.23 * * *$ & & $.19^{* *}$ & & & . \\
\hline
\end{tabular}

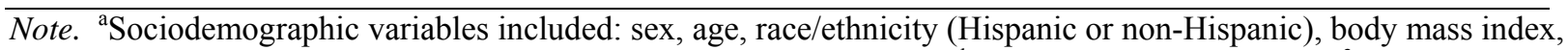
parent's highest education level, income, Verbal IQ, Performance IQ; ${ }^{\mathrm{b}} F(25,217)=2.29, p=.001, R^{2}=.23 ;{ }^{\mathrm{c}} F(25$, $251)=2.67, p<.001, R^{2}=.19 ;{ }^{\mathrm{d}} F(25,251)=2.74, p<.001, R^{2}=.23 ;+p \leq .10 ;{ }^{*} p \leq .05,{ }^{* *} p \leq .01,{ }^{* * *} p \leq .001$ 
Table 8

Hierarchical Regression Results Predicting the Behavior Assessment Scale (BASC-2) for Children-Self-Report of Personality Form (SRP) Social Stress Subscale, the BASC-2 SRP Sense of Inadequacy Subscale, and the BASC-2 SRP Self-Esteem Subscale

\begin{tabular}{|c|c|c|c|c|}
\hline \multirow[t]{2}{*}{ Model } & SRP & $\begin{array}{l}\text { Social } \\
\text { Stress }\end{array}$ & SRP & \multirow{2}{*}{$\begin{array}{c}\text { Sense of } \\
\text { Inadequacy } \\
\beta\end{array}$} \\
\hline & $\Delta \mathrm{R}^{2}$ & $\beta$ & $\Delta \mathrm{R}^{2}$ & \\
\hline Step 1 & .04 & & $.24 * * *$ & \\
\hline \multicolumn{5}{|l|}{ Sociodemographic variables ${ }^{a}$} \\
\hline Step 2 & $.15 * * *$ & & $.16^{* * *}$ & \\
\hline Parent-Reported Insomnia Symptoms (time1) & & -.05 & & .01 \\
\hline Parent-Reported Insomnia Symptoms (time2) & & .06 & & .06 \\
\hline Parent-Reported Daytime Sleepiness (time1) & & .01 & & .03 \\
\hline Parent-Reported Daytime Sleepiness (time2) & & $.14^{*}$ & & $.13^{*}$ \\
\hline Parent-Reported Sleep Duration (time1) & & -.08 & & -.07 \\
\hline Parent-Reported Sleep Duration (time2) & & .03 & & .10 \\
\hline Parent-Reported Weekend Delay (time1) & & .04 & & $.12+$ \\
\hline Parent-Reported Weekend Delay (time2) & & .13 & & .09 \\
\hline Parent-Reported Weekend Oversleep (time1) & & .02 & & -.03 \\
\hline Parent-Reported Weekend Oversleep (time2) & & .02 & & -.00 \\
\hline Self-Reported Insomnia Symptoms & & $.26 * * *$ & & $.19 * * *$ \\
\hline Self-Reported Daytime Sleepiness & & .03 & & .07 \\
\hline Self-Reported Sleep Duration & & -.09 & & $-.19 * *$ \\
\hline Self-Reported Weekend Delay & & -.02 & & .01 \\
\hline Self-Reported Weekend Oversleep & & .01 & & $.14^{*}$ \\
\hline Sleep-Disordered Breathing (time1) & & .07 & & -.03 \\
\hline Sleep-Disordered Breathing (time2) & & .00 & & .05 \\
\hline Total $\mathrm{R}^{2}$ & $.19^{* *}$ & & $.40^{* * *}$ & \\
\hline
\end{tabular}

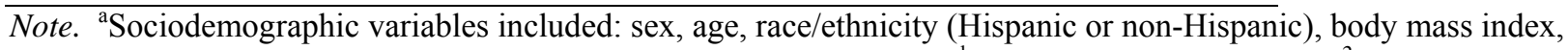
parent's highest education level, income, Verbal IQ, Performance IQ; ${ }^{\mathrm{b}} F(25,251)=2.13, p<.002, R^{2}=.19$; ${ }^{\mathrm{c}} F(25$, $251)=5.92, p<.001, R^{2}=.40 ;+p \leq .10 ;{ }^{*} p \leq .05,{ }^{* *} p \leq .01,{ }^{* * *} p \leq .001$ 